\title{
Re-educating macrophages
}

Despite being the main effectors of the innate immune response and programmed to detect and eliminate mutated cells, macrophages can change their phenotype and become pro-tumorigenic in response to cues from the tumour. Given their position within the tumour mass, tumour-associated macrophages (TAMs) are an attractive therapeutic target, and two new studies have investigated this approach. Guerriero, Nolan, Letai and colleagues pharmacologically reverted the pro-tumorigenic phenotype of TAMs using a class IIa histone deacetylase (HDAC) inhibitor, TMP195, whereas Gholamin, Mitra et al. reactivated the phagocytic activity of macrophages by inhibiting CD47 on tumour cells.

Building on observations that had shown that TMP195 alters C-C motif chemokine ligand 1 (CCL1) and CCL 2 expression in monocytes in response to colony-stimulating factor 1 (CSF1) and promotes a proinflammatory phenotype, Guerriero et al. hypothesized that TMP195 could block differentiation in TAMs. To test this hypothesis, the authors

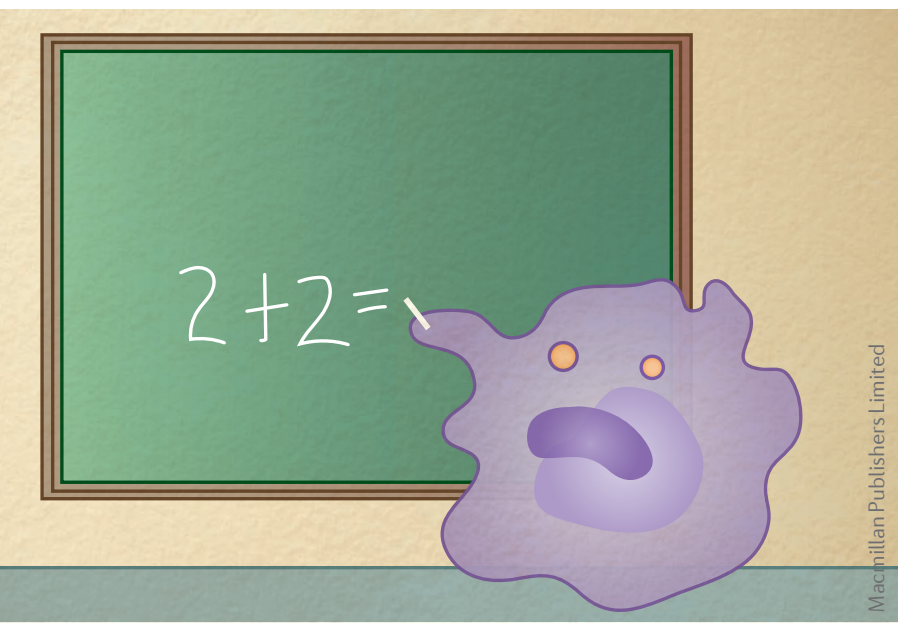

treated MMTV-PyMT transgenic mice - a model of luminal B-type breast cancer in which progression and formation of lung metastasis are regulated by CSF1 and macrophages — with TMP195 for 5 days. Treatment induced an immune response characterized by the activation of CD11 b myeloid cells and mature macrophages in the tumour and a reduction of the proportion of TAMs (that stained positively for CD11b bow and MHC class II). Furthermore, treatment with TMP195 resulted in the recruitment of new macrophages - very few of which were TAMs - and activated cytotoxic T lymphocytes to the tumour. Moreover, the compound improved the integrity of tumour vasculature, increased apoptosis and decreased the proliferation of invasive tumour cells at the leading edge of the tumour.

TMP195 treatment for 13 days significantly reduced the rate of tumour growth, and extending the treatment to 24 days decreased the size and number of metastatic lesions in the lung. This tumour suppression is mediated by activated macrophages and $\mathrm{CD}^{+} \mathrm{T}$ cells, as depletion of myeloid cells or macrophages reduced the antitumor activity of TMP195, and TMP195 failed to inhibit the growth of tumours that had been transplanted into T cell-deficient athymic nude mice. Finally, the authors showed that the antitumour macrophage phenotype induced by TMP195 treatment could enhance the efficacy and durability of both standard chemotherapeutic regimens (carboplatin and paclitaxel) and blockade of the immune checkpoint programmed cell death protein 1 (PD1) in MMTV-PyMT mice.

The anti-phagocytic protein CD47 is overexpressed on the surface of cancer cells, which allows them to evade innate immune surveillance. Blocking the interaction of CD47 with a mouse anti-human CD47 monoclonal antibody (mAb) can inhibit the growth of human tumour cells, so Gholamin, Mitra et al. hypothesized that paediatric brain tumours could be susceptible to CD47 mAbs. They engineered a humanized anti-CD47 $\mathrm{mAb}$ with a human immunoglobulin G4 (IgG4) scaffold (Hu5F9-G4) to minimize the immunogenicity of the antibody. They then tested the antitumour activity of Hu5F9-G4 in human patient-derived primary xenograft models of five aggressive paediatric brain tumours, including medulloblastoma, glioblastoma and diffuse intrinsic pontine glioma. Treatment with Hu5F9-G4 inhibited tumour growth as well as leptomeningeal and spinal metastases, and extended survival by enhancing macrophage recruitment and presence of intratumour macrophages. Importantly, Hu5F9-G4 showed no toxicity against non-malignant human neural cells.

These studies show that the activation of macrophage phagocytosis may not only result in direct therapeutic benefit but can also enhance the efficacy of other regimes. Therefore, it could improve the outcomes in several types of tumours, which underscores the relevance of exploiting the innate immune response in cancer treatment.

\section{Teresa Villanueva}

ORIGINAL ARTICLES Guerriero, J. L. et al. Class Ila HDAC inhibition reduces breast tumours and metastases through anti-tumour macrophages. Nature 543, 428-432 (2017) | Gholamin, S. et al. Disrupting the CD47-SIRPa anti-phagocytic axis by a humanized anti-CD47 antibody is an efficacious treatment for malignant pediatric brain tumors. Sci. Transl. Med. 9, eaaf2968 (2017) FURTHER READING Morrison, C.

Immuno-oncologists eye up macrophage targets. Nat. Rev. Drug Discov. 15, 373-374 (2016) 